\title{
Efficacy of Dietary Interventions on Weight Loss and Biochemical Parameters Among Saudi Females with Obesity and Overweight: An Interventional Non- Randomized Control Trail.
}

Dara Aldisi ( $\nabla$ daldsi_ksu@yahoo.com )

king saud university https://orcid.org/0000-0002-6375-4681

Tayyaba Afsar

King Saud University

Amani Abdullah Al-farraj

King Saud University

Alaa Fahad Alothman

King Saud University

Shajan Hassan Al-Mokyel

King Saud University

Shahad Abdullah AlQahtani

King Saud University

Mariam yousef Bakhsh

King Saud University

Nawarah Ali Alenazy

King Saud University

Suhail Razak

King Saud University

Mona Mohamed Elshafie

King Saud University

Ali Almajwal

King Saud University

\section{Research}

Keywords: Body composition, body fat distribution, obesity, body-mass index, weight Loss, lipids, blood glucose

Posted Date: October 21st, 2020 
DOI: https://doi.org/10.21203/rs.3.rs-93471/v1

License: (c) (1) This work is licensed under a Creative Commons Attribution 4.0 International License. Read Full License 


\section{Abstract}

Background: Numerous dietary approaches have been proposed to treat obesity. We investigated the efficacy of the Ideal Protein Weight Loss Method (IPWLM) diet and the balanced diet (BD) on anthropometric, body composition, and biochemical parameters in obese/overweight subjects.

Methods: 60 overweight/obese females from Riyadh, Saudi Arabia were divided into two groups: Group IPWLM ( $n=29)$ followed the IPWLM diet, while the BD group $(n=31)$ received a balanced diet for 6 weeks.

Result: Significant reduction in anthropometric measurements and body composition were observed in BD and IPWLM groups. In both BD and IPWLM groups, all weight, \% body fat (BF), \% muscle mass, hip and waist circumferences, and body mass index (BMI) had a significant reduction ( $p$, for all trends $<0.05)$. BD group subjects had a significant reduction in triglycerides (TG) and cholesterol $(p<0.05)$, while no changes in HDL and glucose ( $p$, for all trends $>0.05$ ). IPWLM group showed no significant effects on blood glucose and lipid profile (LDL, HDL, and cholesterol ( $p>0.05)$.

Conclusion: Although the IPWLM diet did not improve biochemical parameters, it was significantly associated with improvements in anthropometrics, \%BF, and muscle mass preservation. On the other hand, $\mathrm{BD}$ was more favorable in anthropometrics, body composition as well as biochemical parameters.

\section{Background}

According to the World Health Organization (WHO), overweight and obesity are defined as the abnormal or excessive accumulation of fat that may have deteriorating effects on the health (Organization, 2016). It is well known that excess energy intake is the main cause of obesity. Obesity is a health condition that bears medical, psychological, and economic consequences (Hruby \& Hu, 2015).

The prevalence of obesity in Saudi Arabia is increasing dramatically. "overnight wealth" has created an enormous health burden. Moreover, the current situation has clear indications that this increasing trend would continue (Al-Ghamdi et al., 2018). A previous study revealed the prevalence of obesity in Saudi Arabia as 28.7\% (3.6 million) among Saudi adults, including 24.1\% men and 33.5\% women (Memish et al., 2014). A variety of diets have been proposed to treat obesity. Although, numerous dietary approaches may result in short-term weight loss. Most proposed diets had two main limitations, one limitation is the quick weight regain while another limitation is the lack of long-term compliance by the participant (Abarca-Gómez et al., 2017). One of the dietary approaches to reduce body fat is a balanced diet. This diet ensures adequate nutrient intake and energy balance for weight maintenance and health. As well as the prevention of chronic diseases in healthy individuals. Balanced weight reduction diets restrict energy and stick to the principles of a balance between energy obtained from carbohydrates, fat, and protein, as well as the recommended quality of each macronutrient (Liu et al., 2017).On the other hand, Diets that are high in proteins are becoming more popular. Since 1970 the Ketogenic diet has been used for weight reduction. The ketogenic diet is a low-carbohydrate, high-fat, high-protein diet. A one-year study reported that following a ketogenic diet for the short-term improved many cardiovascular, anthropometric, and 
metabolic parameters related to cardiovascular disease risk (Brown, Buscemi, Milsom, Malcolm, \& O'Neil, 2016; Sumithran, Proietto, \& Practice, 2008). The Ideal Protein Weight Loss Method (IPWLM) is one of the diets that emerged based on the Atkins diet which is the most famous ketogenic diet. The IPWLM is a supplemented ketogenic diet that is expected to show improvements in the anthropometric measurements after following the diet for the short term. The Method has a program of four phases that promotes weight loss. The IPWLM aims to reduce carbohydrates and fats, but not protein. The four-phase program helps participants decrease fat while preserving lean body mass and maintaining the muscles, which is essential for calorie reduction (Masood \& Uppaluri, 2019).

There is no ideal diet that will benefit all individuals suffering from weight regain. Studies have shown controversial evidence regarding the low carbs, high- protein diets. Despite the diet's promising results, few studies addressed the effect of a designed dietary regimen with specific macronutrient content on anthropometric indices (Oh \& Uppaluri, 2019). Body image is becoming an obsession that makes women more willing to follow a different dietary regimen that promises rapid weight loss. Women worldwide are now suffering from the consequences of unhealthy diets (Holland \& Tiggemann, 2016). Dietitians are always looking for new dietary regimens that aim for a healthy weight loss (Trammell, Reed, \& Boylan, 2016). Currently, there is limited evidence regarding the importance of dietary interventions and how diets with specific macronutrient content can affect anthropometric measurements and weight loss. We aimed to examine the effectiveness of the ideal protein weight loss method (IPWLM) diet compared with a balanced diet on anthropometric \& biochemical measurements among overweight and obese females over 6 weeks in Riyadh city. Using include general questionnaire (health profile), pre-designed questionnaire, food frequency questionnaire (FFQ), food records, and anthropometric measurements. This study was challenging it provided a novel and exciting data which will assist dietitians and health practitioners to evaluate and select dietary regimen that will aim for a better weight.

We hypothesized that the IPWLM diet might have a positive effect on anthropometric measurements and biochemical parameters compared to a balanced diet among overweight and obese Saudi females for 6 weeks in Riyadh city. We, therefore, designed this study to investigate the effect of the ideal protein weight loss method (IPWLM) on anthropometric measurements such as (Weight, Height, Body Mass Index, Waist, Hips, Body composition) and biochemical parameters such as (lipid profile and blood glucose) and compared with the effect of balanced diet among overweight and obese females for 6 weeks in Riyadh city. We were specifically interested to study the impact of the ideal protein weight loss method on anthropometric measurements and biochemical parameters.

\section{Methods}

\subsection{Design of intervention}

The number of subjects who were participated in the study is based on the previous study in this area (Te Morenga, Levers, Williams, Brown, \& Mann, 2011). This interventional non-randomized control trial included 60 overweight and obese females aged between (18-50) years for 6 weeks. Participants were 
recruited before obtaining informed consent and screened by the researchers to determine eligibility based on inclusion and exclusion criteria, and then informed consent was signed up from all the subjects who voluntarily agreed to participate in the study. Figure 1 summarized the groups of trials.

Group 1: IPWLM ( $n=31)$ : included participants who followed the Ideal Protein Weight Loss Method (IPWLM) Diet selected from Jwel private Clinic. The disclosure statement of the clinic had been signed by the researchers.

Group 2: BD ( $n=29)$ were recruited from King Saud University campus to receive a balanced diet (BD).

\subsection{Ideal protein weight loss method diet (IPWLM) group}

At the baseline visit subjects were instructed about the diet and how to prepare ideal protein products. Also, were asked to choose as a minimum of three ideal protein foods for breakfast, lunch, dinner, and snack. Food products and supplements (multivitamins, omega3, calcium-magnesium, vitamin D, and potassium) were provided to them with instructions about the allowed food that will be consumed with products which include: 5-8oz of animal protein and 4 cups of low glycemic index vegetables. Supplementary File 1 shows the summary of the dietary recommendations in the IPWLM group.

\subsection{Balanced diet (BD) group}

At the baseline visit, all subjects were provided with information about my plate, food label, fat-free and low-calorie foods, calories and nutritional composition, health consequences of obesity, and related diseases. Supplementary File 2 shows a summary of the dietary recommendations in the BD group. Participants were undergone individual consultation during the intervention program, as well as printed dietary materials and handouts that were designed and provided to all participants to go home according to their assigned dietary regimen to facilitate the implementation of the dietary interventional program. Daily energy requirement (Kcal/day) was estimated using the Harris benedict equation based on age, gender, height, and weight.

The Food record was given to each subject to fill it daily for checking the compliance in the follow-up visit. It included the name and quantity of the food as well as ideal protein products eaten at each meal and snacks, supplements including (multivitamins, calcium, magnesium, zinc, potassium, omega- 3 , and vitamin D), salt intake, water intake, and exercise. As well as printed dietary materials and handouts that were designed and provided to all participants to go home according to their assigned dietary regimen to facilitate the implementation of the dietary interventional program. At the follow-up visit additional FFQ which included examining the intake around the two major food groups (snacks and main meals) was given to subjects. Food groups were defined according to products in the ideal protein website.

\subsection{Primary Screening}


Subjects were screened at King Saud University and Jwel clinic before participation in the study. Where the researcher completed a primary examination of anthropometric measurements and clinical interviews which included questions to determine eligibility based on inclusion and exclusion criteria, detailed health information obtained from all subjects using preset health questionnaire.

\subsection{Exclusion \& inclusion criteria}

Patients with chronic diseases such as cancer, kidney, and liver disease, those on appetite, weight control, and psychology medications, anti-depressant drug and mood stabilizer, vegan, as well as those who participated in weight loss program from three to six months ago, pregnant and lactating, postmenopausal women and subjects under 18 years old, were excluded. All subjects were matched for age, BMI, as closely as possible. Only Saudi females, pre-menopausal, aged between (18-50) years and $\mathrm{BMI} \geq 25 \mathrm{~kg} /$ with normal ECG and blood pressure with no history of vascular disease, renal and liver disease were selected for the study. The infrastructure to undertake these studies forms part of an ongoing research program, and active facilities are available to facilitate this research. These studies were conducted for 6 weeks utilizing only women for this part of the study to increase the compliance rate. The flowchart describing the consort of participants through our clinical trial is presented in Figure 2.

\subsection{Assessment component}

After meeting inclusion and exclusion criteria participants were followed two different types of assigned weight loss methods. Participants were asked to come with overnight fasting (8-10hr) for undergoing a complete assessment. Anthropometric assessment, dietary assessment (food frequency questionnaire, food record), and biochemical assessment. Those measurements were obtained at baseline and at follow up visit after 6 weeks.

\subsection{Anthropometry}

Participating subjects were requested to return to the clinic (at Jwel clinic or King Saud university clinic) for anthropometry. Anthropometric measurements were taken by a well-trained nurse. Anthropometric data were collected with an emphasis on clinical markers of adiposity. Seven variables were evaluated for anthropometric measurement: weight, height, body mass index, waist and hip circumferences, waist to hip ratio, and body composition.

Height was measured by stadiometer while subjects were standing on a flat horizontal surface with their back against the height board. Their heels, buttocks, and shoulder blades should touch the wall or surface of the height board. Before measurements, participants were asked to take a deep breath and hold until the headpiece is pressed against the head. The measurement was read at eye level, to the nearest $0.1 \mathrm{~cm}$ or $0.125 \mathrm{~cm}$. Weight was obtained using the Platform scale. The scale was on a hard, flat, 
horizontal surface calibrated to zero before use. Participants were instructed to wear light clothing, stand over the center of the scale platform, facing front against the scale, without shoes, and with weight evenly distributed between both feet. Weight was determined to the nearest $0.2 \mathrm{Kg}$ and the mean of two variables was taken. (NEBRASKA WIC. 2012)

Waist circumference and Hips were measured when zero ends of the measurement tape are held in one hand and the other end is held in the other hand. Areas have been measured were free of clothing or covered by as little clothing as possible. The tape was snug not tight enough to compress the soft tissue. Measurements were recorded to the nearest $0.1 \mathrm{~cm}$. BMI was calculated as weight $(\mathrm{Kg}) / \mathrm{Height}^{2}\left(\mathrm{~m}^{2}\right)$. Based on BMI, participants were classified as normal, overweight, or obese. Body Composition was assessed using the Tanita body analysis scale. Which gives the whole body composition measurements: weight, percentage of body fat, fat mass, fat-free mass, muscle mass, total body water (TBW), extracellular water (ECW), intracellular water, ECW/TBW ratio, body mass index, bone mass, physique rating, visceral fat rating, basal metabolic rate, and muscle mass balance. All measurements were repeated once post-intervention at 6 weeks.

\subsection{Clinical intervention (dietary regimen)}

For data collection, a pre-designed health questionnaire of Jwel private Clinic was used with some modifications. Some questions were modified, omitted, or added to the questionnaire taking into consideration the participant's understanding. The questionnaire was judged for its face validity through review by three experts in nutrition. The health questionnaire consists of socio-demographic data, medical history, daily habits, and dietary assessment. Participants were assessed two times over a period of 6 weeks. At baseline and follow up visit (after 6 weeks) anthropometric measurements were taken. Health questionnaire and food frequency questionnaires were obtained from subjects in each group at baseline and follow up visit (after 6 weeks) to determine the changes for 6 weeks. Pre-designed Food frequency questionnaire (FFQ) is the dietary tool that was used to assess the food intake of the subjects prior and post the dietary intervention (Al-Daghri et al., 2015; Al-Disi et al., 2010). The FFQ includes examining the intake around the five major food groups (starch, grains, meat \& beans, dairy products, fruits, and vegetables) along with the consumption of the junk food which are defined as heavily processed, highly palatable, and hyper-energetic and are often deprived of the vitamins and essential nutrients found in whole unprocessed foods (Figure 3) since it poses the greatest risk to health and wellbeing (Blackburn, 2001); energy-dense, nutrient-poor products were also included illustrated through the consumption of fat (cream, ghee, and nuts), sweets (cakes, honey, jam, ice-creams, donuts, and chocolates), salty snacks (pop-corn), soft drinks and caffeinated drinks (coffee, black and green tea(. Also, contains Ideal protein meal replacement products.

The researcher interviewed the participants individually and the information was collected using FFQ to assess the qualitative and the quantitative aspects of the food consumed by the participants for 6 weeks 
to check for compliance. The health questionnaire was given again to all subjects to assess changes for 6 weeks.

All participants have received health advice according to their assigned type of diet and were interviewed individually. To support compliance health advice was confirmed by phone messages or E-mail (once/week), Participant was asked to record all food eaten daily for 6 weeks. Food records were collected by the researcher and returned with feedback. Food record for group IPWLM included the name and quantity of food, ideal protein products eaten at each meal and snacks, supplements including (multivitamins, calcium, magnesium, zinc, potassium, omega-3, and vitamin D), salt intake, water intake, and exercise. The food record for group BD included the quantity and component of food eaten in each meal and snacks, details about ready to eat products and drinks.

Nutrient intake was calculated using the USDA database (18th- 21st Ed, 2009, 2010, Program, ars.www.usda.gov), as for the Saudi Arabic traditional dishes were analyzed using the Arabic food analysis program (version 1,2006). The evaluation of daily food intake was made by the means of total energy and the total nutrient intake, the percentage of the total calories derived from fat, protein, and carbohydrates. Dietary nutrients values were compared with dietary reference intake (DRI) for specified age and gender for macronutrients (carbohydrates and protein) as well as for micronutrients (DRI, 2008/2011, Supplementary file 3).

\subsection{Blood sample collection}

The blood samples were collected using sterile vacutainer blood collection apparatus. Green/Gray mottled Plasma Separator Tube (PST) with heparin was used. All samples were aliquot and stored in $20 . \mathrm{C}$ freezer facilities in preparation for subsequent analysis by spectrophotometer. A spectrophotometer consists of two instruments, namely a spectrometer for producing light of any selected color (wavelength), and a photometer for measuring the intensity of light. The instruments are arranged so that liquid in a cuvette can be placed between the spectrometer beam and the photometer. The amount of light passing through the tube is measured by the photometer. The photometer delivers a voltage signal

to a display device, normally a galvanometer. The signal changes as the amount of light absorbed by the liquid changes. If the development of color is linked to the concentration of a substance in solution, then that concentration can be measured by determining the extent of absorption of light at the appropriate wavelength.

\subsection{Biochemical assessment}

Blood samples from all subjects were analyzed for various biochemical parameters. blood glucose and lipid profiles (total cholesterol, HDL, and LDL) were determined using standard AMP diagnostic kits (Stattogger Strasse 31b 8045 Graz, Austria). The instrument was calibrated before analysis using quality 
control samples provided with the kits. The parameters were measured at baseline and follow up visits after 6 weeks for all groups.

\subsection{Statistical analysis}

Data were analyzed using (SPSS) version 22 (SPSS, Chicago, IL, USA). Power was used for the calculation of sample size ascertaining the difference between two dependent means (matched pairs). A final sample size of 60 with $a=0.05$ and an effect size of 0.0 .651 has a power of 0.200 to detect the difference. All variables are present as mean \pm standard deviation. For comparison between baseline and post-intervention, a paired t-test has been used. For comparison between groups (IPWLM group, and BD group), a randomized sample of 15 subjects was selected from the BD group to minimize the variation in sample size between groups and an independent sample t-test has been used. Significance was set at $\mathrm{p} \Downarrow$ 0.05 .

\section{Results}

\subsection{General characteristics at Baseline}

Initially, 74 subjects agreed to participate. However, 14 subjects dropped out at various stages of the study. Therefore, data could be completed on a total of 60 subjects. Table 1 shows the general characteristics of subjects at baseline. Data on mean age shows subjects in BD were older than subjects in the IP group (mean age 21.87 vs. 44.56 years). Subjects in the two groups were almost similar based on other parameters.

\subsection{Effects on anthropometric measurements and body composition}

Table 2 shows mean (std) differences in anthropometric measurements and body composition in BD and IP groups after intervention. The results showed a significant reduction in anthropometric measurements and body composition in $\mathrm{BD}$ and IP groups. In the $\mathrm{BD}$ group, weight, \%BF, \% muscle mass, hip and waist circumferences, and BMI had a significant reduction ( $p$, for all trends $<0.05$ ). In the IP group, weight, BMI, waist circumference, and \% BF were reduced significantly ( $p$, for all trends $<0.05$ ). However, a nonsignificant reduction in WHR ( $p$-value $=0.191)$, muscle\% $(p$-value $=0.220)$ were observed in subjects in IP group.

\subsection{Effects on Biochemical parameters}

Table 3 shows mean (std) differences in selected biochemical in subjects in BD and IP groups after the intervention. In subjects in the BD group, a significant reduction was observed in TG and cholesterol $(p$, 
for all trends $<0.05$ ), but there was no significant effect on HDL and glucose ( $p$, for all trends $>0.05)$. on the contrary, there were no significant effects in biochemical parameters in the IP group ( $p$, for all trends $>0.05)$.

\section{Discussion}

In the present study, we examined the impact of the ideal protein weight loss method diet (IPWLM) and the balanced weight reduction diet (BD) on anthropometric measurements and biochemical parameters.

One of the scientific dietary approaches to reduce body weight is a balanced diet. This diet ensures adequate nutrient intake and energy balance for weight maintenance and health. As well as the prevention of chronic diseases in healthy individuals. Balanced weight reduction diets restrict energy and stick to the principles of a balance between energy obtained from carbohydrates, fat, and protein, as well as the recommended quality of each macronutrient (Koliaki et al., 2018). There are few studies conducted worldwide to evaluate the effect of a balanced diet on weight management in healthy obese individuals, while considering many factors such as gender, BMI, age, and race (Rondanelli et al., 2015). The Ideal Protein Weight Loss Method (IPWLM) is a ketogenic supplemented diet that is suspected to reduce weight and preserve the body's muscle and reducing fat mass. Studies have shown controversial evidence regarding low carbs, high- protein diets (Paoli, Rubini, Volek, \& Grimaldi, 2013). The study found that a balanced diet showed a reduction in the weight, waist, hip, fat\%, BMI, waist-to-hip ratio. Muscle percentage varied between participants. Results of the biochemical parameters in the BD group showed various effects. The reduction was seen in Triglyceride and cholesterol. While, HDL and glucose were not affected.

Our results appear to demonstrate that the (IPWLM), did not improve the biochemical parameters however significant weight loss was observed. On contrary, previous reports revealed rapid weight loss and marked improvement in biochemical parameters in specific protein diet users in a short period (www.medaxiom.com) (Baer et al., 2011; Urbain et al., 2017). In the current intervention, we observed that IPWLM was associated with appreciable reductions in weight, fat percentage, waist, and hip circumferences and BMI. The favorable changes occurred without any loss of lean body mass. On the other hand, no effect was recorded on the waist-to-hip ratio and muscle percentage. There were no significant differences between the two groups in the anthropometric measurements and biochemical parameters as shown in Table 1. Whilst it was already anticipated that there would be weight loss on such diets it was interesting to observe how dietary modifications could also improve the anthropometric and biochemical measurements and overall health of subjects (Calton, James, Pannu, \& Soares, 2014; McAdam-Marx et al., 2014; Webb, 2019).

Although the study may seem relatively simple in outlook, there is a severe scarcity of dietary intervention studies in the region, specifically in Saudi Arabia, where most prospective studies focus on the effects of health during the holy month of Ramadan which has nothing to do with caloric restriction (Abazid et al., 2018; Trepanowski \& Bloomer, 2010). Furthermore, most of the other studies in the region appear to 
provide review analysis or cross-sectional studies on the dietary habits of the population (Abulmeaty et al., 2019; Al-Daghri et al., 2013; Alam et al., 2019). Thus these studies were undertaken to provide the first pieces of evidence that such dietary intervention can work in Saudi culture to fill the gaps in existing knowledge. Interestingly, the responses of both the anthropometric and biochemical parameters varied depending on the type of dietary intervention provided (ideal protein versus balanced weight reduction diet), with the balanced diet being more favorable in both anthropometric \& biochemical parameters. The ideal protein method was more compatible with preserving the muscle mass.

\section{Study Limitations}

The intervention was for relatively a short time ( 6 weeks) and most of the dependent variables might have been affected if the intervention was for at least one year. Glucose was not affected in both diets due to the time limitation, a longer study period may have provided greater insight into the effects of the diets. Another study limitation is the limited university resources and small sample size as well as the cost of the ideal protein weight loss method. Despite all these limitations, this study is the ever first study conducted in the Middle East, and future studies to follow should address all these limitations in their study designs.

\section{Conclusion}

Our results appear to demonstrate that although the IPWLM diet, did not improve the biochemical parameters, it was associated with appreciable reductions in anthropometric measurements, fat percentage while the favorable changes occurred with muscle's mass preservation. On the other hand, the balanced diet is more favorable in both anthropometric \& biochemical parameters. More research is required in this area. Further investigations examining the effects of different types of Diets on anthropometric measurements and biochemical data are required. Our research gives insight and awareness among the population to choose the effective weight loss strategies that not are not only effective in terms of weight loss but are beneficial for overall health because obesity leads to various other idiosyncratic disorders so planning a balanced diet will lead to maintaining overall health as well as reducing the weight. Future studies should explore whether these findings can be more significant if done on a larger population and a longer period. Other population groups such as males also need to be examined to determine their response to the diets and to be able to generalize the results on the whole population. More detailed information about the ideal protein IPWLM effect on an individual's health and biochemical markers are needed.

\section{List Of Abbreviations}

IPWLM: Ideal protein weight loss method

BD: Balanced diet 
BF: Body fat

BMI: Body mass index

TG: Triglycerides

HDL: High-density lipoproteins

LDL: Low-density lipoproteins

TBW: Total body water

ECW: Extracellular water

ECG: Electrocardiogram

DRI: Dietary reference intake

PST: Plasma Separator Tube

FFQ: Food Frequency questionnaire

\section{Declarations}

\section{Ethical Approval}

Ethical approval (CAMS 035-37/38) for the current study was provided by the Ethics Committee of applied medical sciences, at King Saud University, Riyadh, Kingdom of Saudi Arabia, before the commencement of the research.

\section{Consent to participate}

Not applicable

\section{Consent for publication}

Not applicable

\section{Availability of supporting data}

The datasets used and/or analyzed during the current study are available from the corresponding author on reasonable request.

\section{Competing interests}

The authors proclaim that they have no competing interests. 


\section{Funding}

We are grateful to the Deanship of Scientific Research at King Saud University for its funding of this research through Research Group Project number 193.

\section{Authors' contributions}

DA is the PI of the study and made a major contribution to the design and conception of the research. TA and SR wrote the manuscript. AAF, AFO, SHA, SAQ, MYB, and NAA collected the data, perform experimentation, analyses the data, and wrote the methodology, results, and discussion. MME, SR, and AA provide significant assistance in revising the manuscript for intellectual content. All authors read and accepted the final manuscript.

\section{Acknowledgments}

We are grateful to the Deanship of Scientific Research at King Saud University for its funding of this research through Research Group Project number 193.

\section{References}

1. Abarca-Gómez, L., Abdeen, Z. A., Hamid, Z. A., Abu-Rmeileh, N. M., Acosta-Cazares, B., Acuin, C., . . . Aguilar-Salinas, C. A. J. T. L. (2017). Worldwide trends in body-mass index, underweight, overweight, and obesity from 1975 to 2016: a pooled analysis of 2416 population-based measurement studies in 128. 9 million children, adolescents, and adults. 390(10113), 2627-2642.

2. Abazid, R. M., Khalaf, H. H., Sakr, H. I., Altorbak, N. A., Alenzi, H. S., Awad, Z. M., . . Azazy, A. S. J. S. m. j. (2018). Effects of Ramadan fasting on the symptoms of chronic heart failure. 39(4), 395.

3. Abulmeaty, M., Almajwal, A. M., ElSadek, M. F., Aldisi, D., Al-Momani, M. M., Alquraishii, M., ... Almutawa, D. A. J. P. I. N. (2019). Dietary and lifestyle determinants of the lifetime cardiovascular risk during early adulthood. 21, 297-306.

4. Al-Daghri, N. M., Al-Attas, O., Yakout, S., Aljohani, N., Al-Fawaz, H., Alokail, M. S. J. I. j. o. c., \& medicine, e. (2015). Dietary products consumption in relation to serum 25-hydroxyvitamin D and selenium level in Saudi children and adults. 8(1), 1305.

5. Al-Daghri, N. M., Khan, N., Alkharfy, K. M., Al-Attas, O. S., Alokail, M. S., Alfawaz, H. A., . . Vanhoutte, P. M. J. N. (2013). Selected dietary nutrients and the prevalence of metabolic syndrome in adult males and females in Saudi Arabia: a pilot study. 5(11), 4587-4604.

6. Al-Disi, D., Al-Daghri, N., Khanam, L., Al-Othman, A., Al-Saif, M., Sabico, S., \& Chrousos, G. J. E. j. (2010). Subjective sleep duration and quality influence diet composition and circulating adipocytokines and ghrelin levels in teen-age girls. 1008090469-1008090469.

7. Al-Ghamdi, S., Shubair, M. M., Aldiab, A., Al-Zahrani, J. M., Aldossari, K. K., Househ, M., . . disease. (2018). Prevalence of overweight and obesity based on the body mass index; a cross-sectional study in Alkharj, Saudi Arabia. 17(1), 134. 
8. Alam, I., Alam, W., Aljuraiban, G. S., Abulmeaty, M., Shivappa, N., Razak, S. J. N., \& cancer. (2019). Nutritional, immunological and antioxidant defense status of outpatients diagnosed with colorectal cancer-a case-control study of the little-studied population. 1-14.

9. Baer, D. J., Stote, K. S., Paul, D. R., Harris, G. K., Rumpler, W. V., \& Clevidence, B. A. J. T. J. o. n. (2011). Whey protein but not soy protein supplementation alters body weight and composition in free-living overweight and obese adults. 141(8), 1489-1494.

10. Blackburn, G. L. J. O. r. (2001). Treatment approaches: food first for weight management and health. 9(S11), 223S-227S.

11. Brown, J. D., Buscemi, J., Milsom, V., Malcolm, R., \& O'Neil, P. M. J. T. b. m. (2016). Effects on cardiovascular risk factors of weight losses limited to 5-10\%. 6(3), 339-346.

12. Calton, E. K., James, A. P., Pannu, P. K., \& Soares, M. J. J. N. r. (2014). Certain dietary patterns are beneficial for the metabolic syndrome: reviewing the evidence. 34(7), 559-568.

13. Holland, G., \& Tiggemann, M. J. B. i. (2016). A systematic review of the impact of the use of social networking sites on body image and disordered eating outcomes. 17, 100-110.

14. Hruby, A., \& Hu, F. B. J. P. (2015). The epidemiology of obesity: a big picture. 33(7), 673-689.

15. Koliaki, C., Spinos, T., Spinou, M., Brinia, M.-E., Mitsopoulou, D., \& Katsilambros, N. (2018). Defining the optimal dietary approach for safe, effective and sustainable weight loss in overweight and obese adults. Paper presented at the Healthcare.

16. Liu, A. G., Ford, N. A., Hu, F. B., Zelman, K. M., Mozaffarian, D., \& Kris-Etherton, P. M. J. N. j. (2017). A healthy approach to dietary fats: understanding the science and taking action to reduce consumer confusion. 16(1), 53.

17. Masood, W., \& Uppaluri, K. R. (2019). Ketogenic Diet. In StatPearls [Internet]. StatPearls Publishing.

18. McAdam-Marx, C., Bellows, B. K., Unni, S., Wygant, G., Mukherjee, J., Ye, X., \& Brixner, D. I. J. J. o. M. C. P. (2014). Impact of adherence and weight loss on glycemic control in patients with type 2 diabetes: cohort analyses of integrated medical record, pharmacy claims, and patient-reported data. 20(7), 691-700.

19. Memish, Z. A., El Bcheraoui, C., Tuffaha, M., Robinson, M., Daoud, F., Jaber, S., . . Mokdad, A. H. J. P. c. d. (2014). Peer reviewed: obesity and associated factors-Kingdom of Saudi Arabia, 2013. 11.

20. Oh, R., \& Uppaluri, K. R. (2019). Low Carbohydrate Diet. In StatPearls [Internet]: StatPearls Publishing.

21. Organization, W. H. (2016). Consideration of the evidence on childhood obesity for the Commission on Ending Childhood Obesity: report of the ad hoc working group on science and evidence for ending childhood obesity, Geneva, Switzerland.

22. Paoli, A., Rubini, A., Volek, J., \& Grimaldi, K. J. E. j. o. c. n. (2013). Beyond weight loss: a review of the therapeutic uses of very-low-carbohydrate (ketogenic) diets. 67(8), 789-796.

23. Rondanelli, M., Giacosa, A., Faliva, M. A., Perna, S., Allieri, F., \& Castellazzi, A. M. J. W. J. o. C. C. W. (2015). Review on microbiota and effectiveness of probiotics use in older. 3(2), 156. 
24. Sumithran, P., Proietto, J. J. O. R., \& Practice, C. (2008). Ketogenic diets for weight loss: a review of their principles, safety and efficacy. 2(1), 1-13.

25. Te Morenga, L. A., Levers, M. T., Williams, S. M., Brown, R. C., \& Mann, J. J. N. j. (2011). Comparison of high protein and high fiber weight-loss diets in women with risk factors for the metabolic syndrome: a randomized trial. 10(1), 40.

26. Trammell, E. L., Reed, D., \& Boylan, M. J. T. i. C. N. (2016). Education and practice gaps of registered dietitian nutritionists working with clients with eating disorders. 31(1), 73-85.

27. Trepanowski, J. F., \& Bloomer, R. J. J. N. j. (2010). The impact of religious fasting on human health. 9(1), 57.

28. Urbain, P., Strom, L., Morawski, L., Wehrle, A., Deibert, P., Bertz, H. J. N., \& metabolism. (2017). Impact of a 6-week non-energy-restricted ketogenic diet on physical fitness, body composition and biochemical parameters in healthy adults. 14(1), 17.

29. Webb, G. P. (2019). Nutrition: maintaining and improving health: CRC Press.

\section{Tables}


Table 1:

Characteristics of the trial participants at baseline between groups

\begin{tabular}{|c|c|c|c|c|}
\hline Parameters & Group* & Mean & Std. Deviation & Std. Error Mean \\
\hline \multirow[t]{2}{*}{ Age (yrs) } & $\mathrm{BD}$ & 21.87 & 2.748 & 0.710 \\
\hline & IP & 44.56 & 7.923 & 2.641 \\
\hline \multirow[t]{2}{*}{ Weight (Kg) } & $\mathrm{BD}$ & 81.473 & 16.2657 & 4.1998 \\
\hline & IP & 81.844 & 11.3040 & 3.7680 \\
\hline \multirow[t]{2}{*}{ Height (cm) } & $\mathrm{BD}$ & 161.560 & 6.5009 & 1.6785 \\
\hline & IP & 160.222 & 6.8880 & 2.2960 \\
\hline \multirow[t]{2}{*}{ BMI (Kg/m2) } & $\mathrm{BD}$ & 31.060 & 5.2149 & 1.3465 \\
\hline & IP & 31.811 & 3.6597 & 1.2199 \\
\hline \multirow[t]{2}{*}{ Waist Circumference (cm) } & $\mathrm{BD}$ & 91.033 & 12.2335 & 3.1587 \\
\hline & IP & 88.444 & 7.4517 & 2.4839 \\
\hline \multirow[t]{2}{*}{ Hip Circumference (cm) } & $\mathrm{BD}$ & 116.087 & 9.3399 & 2.4116 \\
\hline & IP & 110.444 & 7.8280 & 2.6093 \\
\hline \multirow[t]{2}{*}{ WHR } & $\mathrm{BD}$ & .7813 & .06116 & .01579 \\
\hline & IP & .7967 & .03279 & .01093 \\
\hline \multirow[t]{2}{*}{ BF (\%) } & $\mathrm{BD}$ & 42.7600 & 5.64596 & 1.45778 \\
\hline & IP & 40.0000 & 4.23615 & 1.41205 \\
\hline \multirow[t]{2}{*}{ Muscle (\%) } & $\mathrm{BD}$ & 53.2467 & 4.62383 & 1.19387 \\
\hline & IP & 56.5511 & 3.94322 & 1.31441 \\
\hline \multirow[t]{2}{*}{ Blood Glucose } & $\mathrm{BD}$ & 98.8667 & 5.14492 & 1.32841 \\
\hline & IP & 101.5393 & 4.33320 & 1.44440 \\
\hline \multirow[t]{2}{*}{ TG } & $B D$ & 1.9309 & .08692 & .02244 \\
\hline & IP & 1.5447 & .19855 & .06618 \\
\hline \multirow[t]{2}{*}{ Cholesterol } & $\mathrm{BD}$ & 4.0673 & .25416 & .06562 \\
\hline & IP & 5.7139 & .32604 & .10868 \\
\hline \multirow[t]{2}{*}{ HDL } & BD & 78.4040 & 11.32003 & 2.92282 \\
\hline & IP & 67.3711 & 11.14173 & 3.71391 \\
\hline
\end{tabular}


*BD=Balanced diet group; IP= Ideal Protein Weight Loss Method (IPWLM) Diet group; WHR= waist to hip ratio; $\mathrm{TG}=$ Triglycerides; $\mathrm{HDL}=$ high density lipoprotein

Table 2:

Anthropometric and body composition measurements in intervention vs control groups

\begin{tabular}{|lll|}
\hline Parameters & $\begin{array}{l}\text { Mean Difference between the values before intervention and after } \\
\text { intervention }\end{array}$ \\
\hline BD $(\mathrm{n}=31)$ & IP $(\mathbf{n}=\mathbf{2 9})$ \\
\hline Weight $(\mathrm{kg})$ & $1.29(2.1)^{\star}$ & $2.37(1.4)^{\star \star}$ \\
\hline BMI $\left(\mathrm{kg} / \mathrm{m}^{2}\right)$ & $0.44(0.84)^{\star}$ & $0.85(0.4)^{\star \star}$ \\
\hline $\begin{array}{l}\text { Waist circumference } \\
(\mathrm{cm})\end{array}$ & $3.28(5.1)^{\star}$ & $2.33(1.4))^{\star \star}$ \\
\hline Hip circumference $(\mathrm{cm})$ & $1.61(3.1)^{\star}$ & $3.44(1.3)^{\star \star}$ \\
\hline WHR & $0.02(0.03)^{\star}$ & $0.05(0.01)$ \\
\hline BF $(\%)$ & $0.98(1.5)^{\star *}$ & $1.50(1.2)^{\star}$ \\
\hline Muscle\% & $1.03(1.8)^{\star}$ & $11.06(24.9)$ \\
\hline
\end{tabular}

1 differences in mean values for anthropometric and body composition measurements were determined by subtracting values after intervention form values at baseline

*=significant $p<0.05$

$\star *=$ very significant at $p<0.01$

Table 3:

Biochemical profile in intervention vs control groups

\begin{tabular}{|lll|}
\hline $\begin{array}{l}\text { Biochemical } \\
\text { parameters }\end{array}$ & $\begin{array}{l}\text { Mean Difference between the values before intervention and after } \\
\text { intervention }\end{array}$ \\
\hline & $\mathrm{BD}(\mathrm{n}=31)$ & $\mathrm{IP}(\mathrm{n}=29)$ \\
\hline Glucose & $1.80(5.54)$ & $4.28(14.82)$ \\
\hline TG & $0.43(0.15)^{\star \star}$ & $0.04(12.1)$ \\
\hline Cholesterol & $1.10(0.52)^{\star \star}$ & $1.17(1.81))$ \\
\hline HDL & $2.04(18.1)$ & $1.41(13.6)$ \\
\hline
\end{tabular}

${ }^{1}$ differences in mean values for anthropometric and body composition measurements were determined by subtracting values after intervention form values at baseline

*=significant at $p<0.05$ 
$\star \star=$ very significant at $p<0.01$

$\mathrm{BD}=$ Balanced diet group; IP= Ideal Protein Weight Loss Method (IPWLM) Diet group; TG=Triglycerdis; $\mathrm{HDL}=$ high density lipoprotein

\section{Figures}

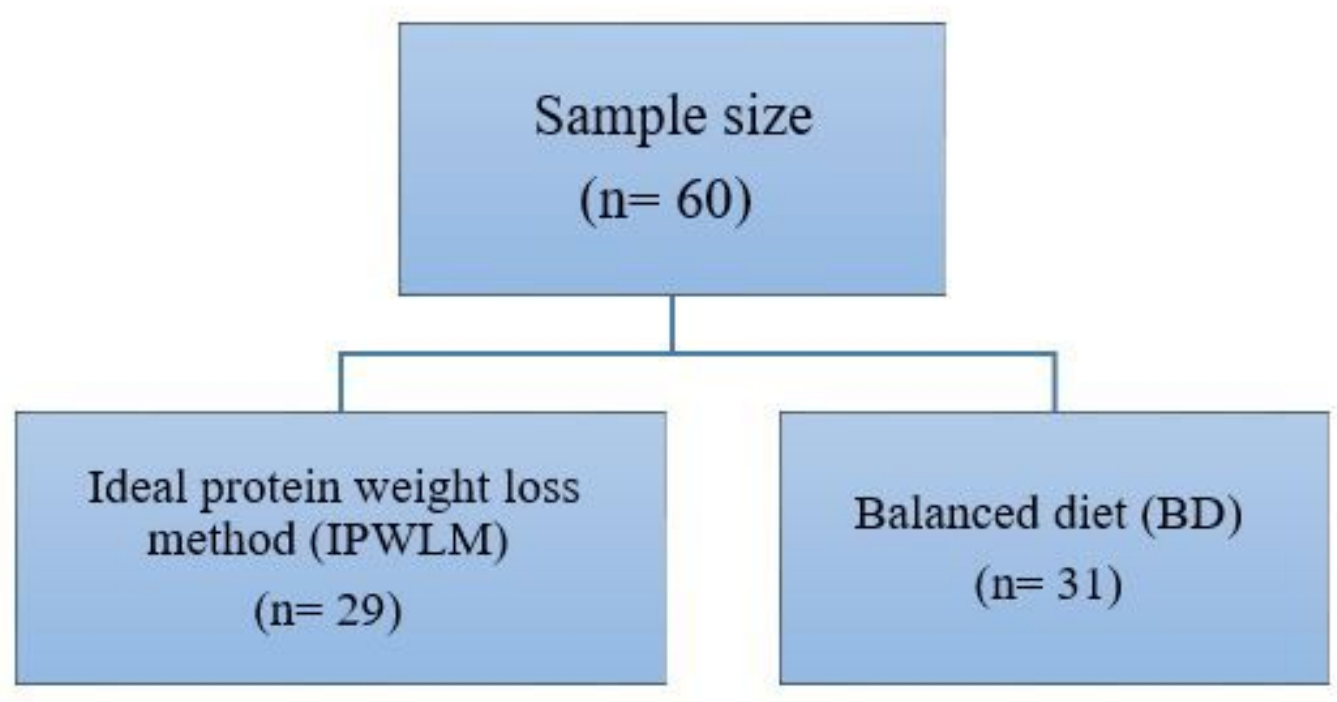

Figure 1

Arms of the interventional trial proposed: Subjects were divided into two groups according to their assigned type of dietary regimen (IPWLM = Ideal protein weight loss method) group and (BD =Balanced diet) group 


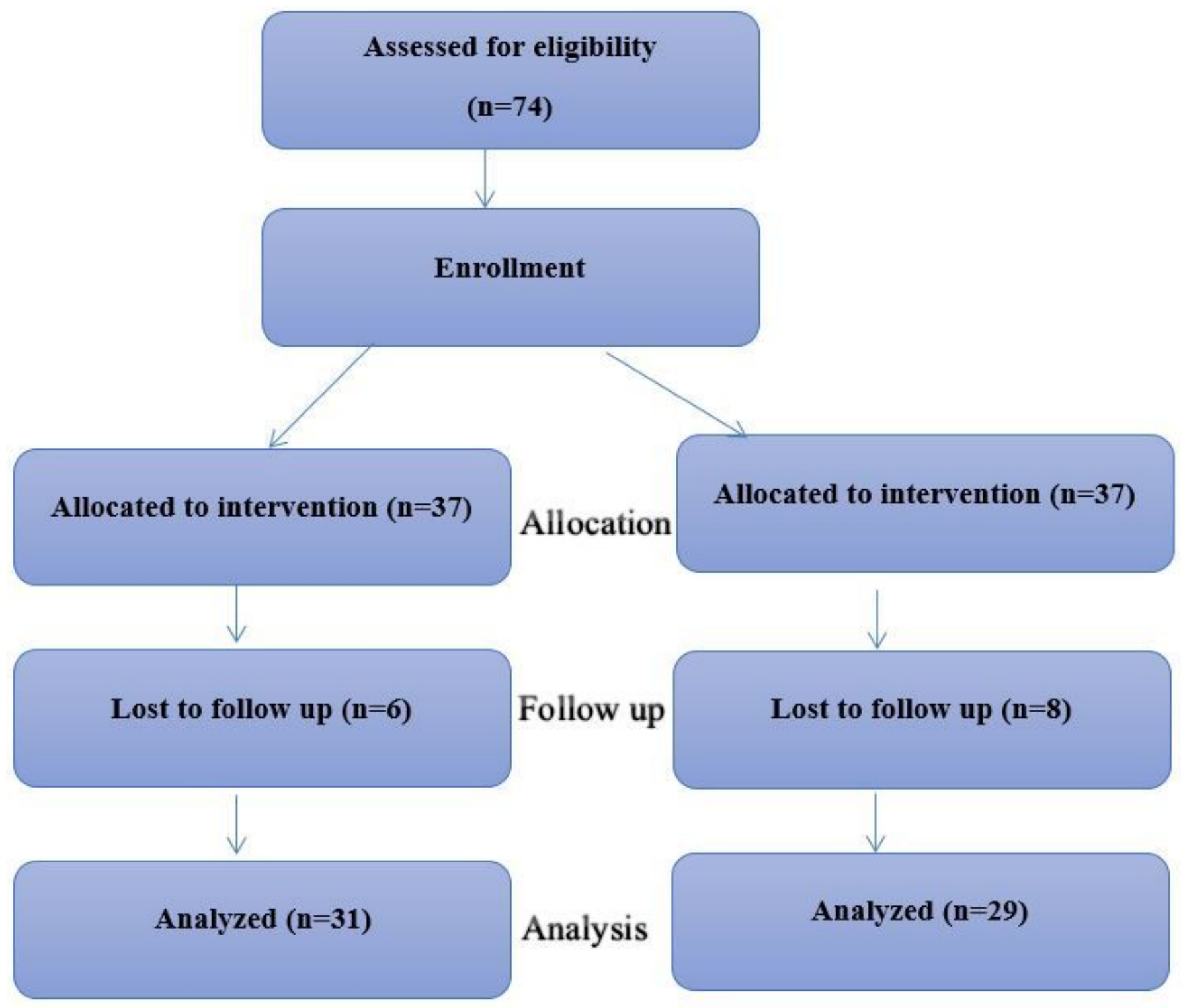

Figure 2

Flowchart describing the consort of participants through our trial 


\section{HEALTHY EATING PLATE}

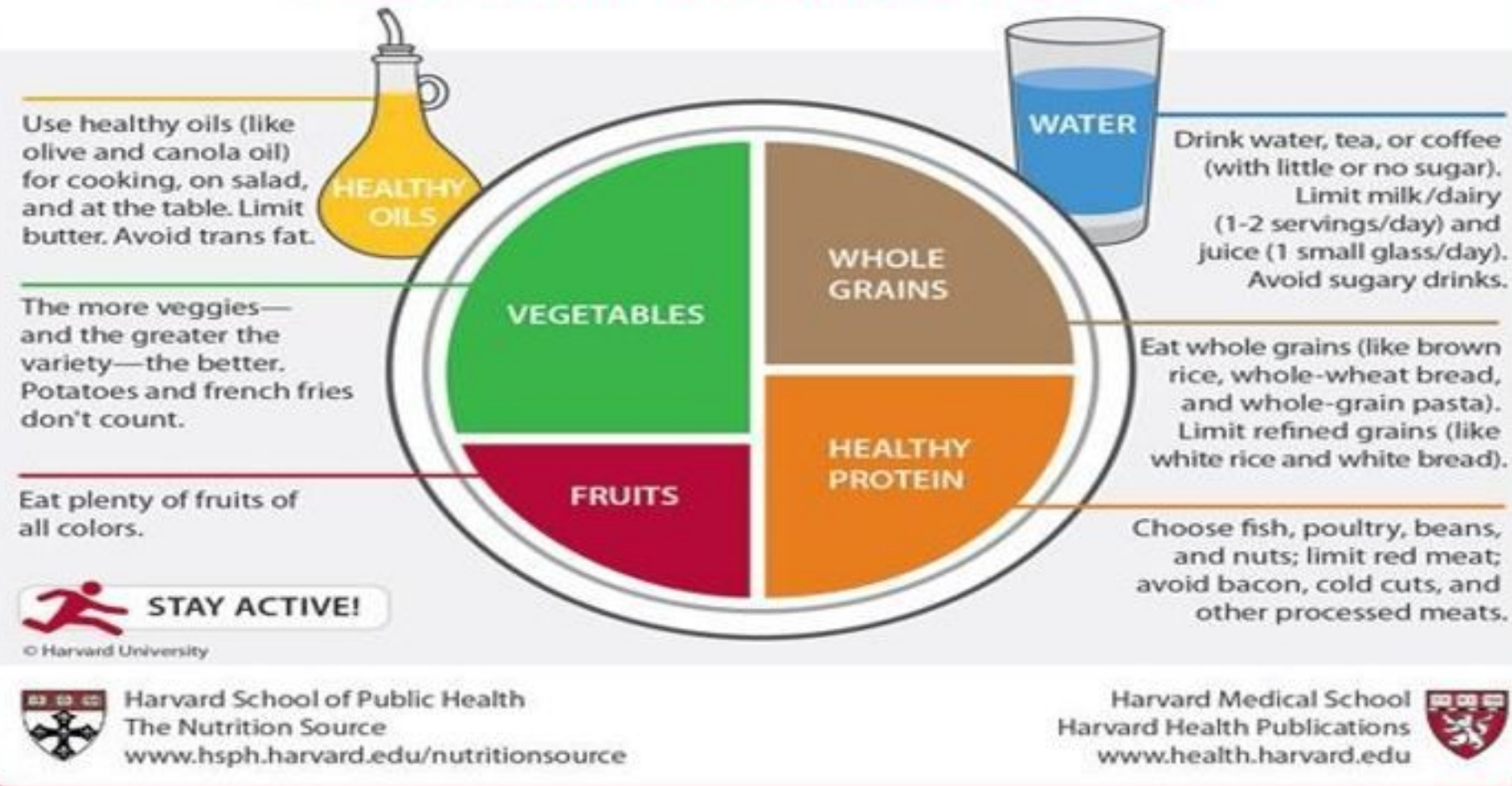

Figure 3

The healthy eating plate helps to create healthy \& flavorful meals. Created by nutrition experts at Harvard School of Public Health in conjunction with Harvard Health Publications, The Healthy Eating Plate addresses key flaws in the U.S. Department of Agriculture's MyPlate (www.hsph.harvard/nutritionsource).

\section{Supplementary Files}

This is a list of supplementary files associated with this preprint. Click to download.

- Supplemetaryfile3.docx

- Supplimentaryfile2.docx

- supplimentayfile1.docx 